\title{
SENSITIVITY STUDY FOR A 4-ELECTRODE FOCUSED IMPEDANCE METHOD (FIM) USING FINITE ELEMENT METHOD ANALYSIS
}

\author{
Ahmed Raihan Abir* and K Siddique-e Rabbani \\ Department of Biomedical Physics \& Technology, University of Dhaka, Dhaka 1000, Bangladesh \\ *Corresponding author email: raihan1079@gmail.com
}

Received: 23 December 2013; Accepted:25 July 2014

\begin{abstract}
Focused Impedance Method (FIM) is a recent technique, innovated by one of the authors at Dhaka, and shows a promise in providing practical results with a minimum of instrumentation complexity. Out of three version using 8, 6 and 4 electrodes, this paper concentrates on the 4-electrode FIM. Earlier work mainly used phantom experiments to verify the concept and analytical methods for a semi-infinite medium. The present work uses Finite Element Method (FEM), implemented by a software package named COMSOL to obtain various sensitivity graphs for 4-FIM. Firstly, it has verified the focusing effect for a homogenous medium using point sensitivity calculations. Secondly, it obtained simulated impedances for finite sized objects embedded in a medium of uniform conductivity considering five important variables namely, electrode spacing, object size, object resistivity, background resistivity and depth. In this paper the effect of each of these variables has been studied by keeping the other four constant. The obtained graphs give an insight which is expected to lead to quantitative assessment of target objects, needed in physiological study and diagnosis.
\end{abstract}

\section{INTRODUCTION}

Focused Impedance Method is attractive because of its ability to localize a small region in a volume conductor using a few electrodes and simple instrumentation [Rabbani et al 1999, Rabbani and Karal 2008, Islam et al, 2010, Rabbani 2010]. Utilising the 3D sensitivity of FIM it is possible to detect objects at shallow depths using skin surface electrodes, typically of the order of the separation of the electrodes [Iquebal and Rabbani 2010]. FIM has potential applications in many physiological studies and in the detection and diagnosis of diseases and disorders of the human body [Rabbani and Kadir 2011]. Particularly it may be useful in studying stomach emptying, stomach acid secretion, localized lung ventilation [Kadir 2010], bladder emptying, etc., where temporal changes occur that may be discerned through a difference measurement. Again, through changes in the frequency of measurement it may be possible to identify organs or localized tissues that have distinctive frequency spectrum from the surrounding tissues as has been done in the detection of cervical cancer by a group in Sheffield using a slightly different method. Thus it may be possible to characterise breast tumours and to monitor tumour ablation at shallow depths using multiple frequency measurements.

Out of the three versions developed, with 8,6 and 4 electrodes respectively, the 4 electrode FIM (abbreviated as 4-FIM) is simple in electrode configuration and useful in many applications. The concept was initially developed with the help of equipotential lines as shown in Figure 1. Here 4 electrodes, 1,2,3,4 are fixed in a square formation. Firstly, current is driven through electrodes $1 \& 2$ while potential is measured across electrodes $3 \& 4$. This gives a transfer impedance $Z_{1}$ which is essentially due to the impedance of the horizontal shaded region between the equipotential lines passing through electrodes $3 \& 4$. Then current is driven through electrodes $2 \& 3$ while potential is measured across electrodes $4 \& 1$. This gives a transfer impedance $Z_{2}$ which is essentially due to the impedance of the vertical shaded region. The Focused Impedance FZ is defined as the average of the two impedances. 


$$
\mathrm{FZ}=\left(\mathrm{Z}_{1}+\mathrm{Z}_{2}\right) / 2
$$

The concept of FIM was initially verified through carefully conducted phantom experiments and through real life stomach emptying and lungs ventilation studies which appeared to show expected behaviours [Rabbani et al 1999]. Later analyses based on lead field theorems and implemented using MATLAB demonstrated the focusing effect although near the electrode planes large spiky sensitivity were observed at the electrodes [Islam et al 2010].

FIM is a system based on traditional tetrapolar impedance measurement (TPIM) which uses two electrodes for driving a current and two electrodes to pick up the resulting potential. Any TPIM has an associated negative sensitivity in a volume conductor in the regions between the respective current and potential electrodes. This means that if an object of high electrical impedance is placed in these regions within a volume conductor of uniform conductivity, the measured transfer impedance will show a decreased value compared to that without the object. This is in contrast to a positive sensitivity within the two potential electrodes, the target measuring zone, which will give an increased value of measured transfer impedance if the same object is placed there.

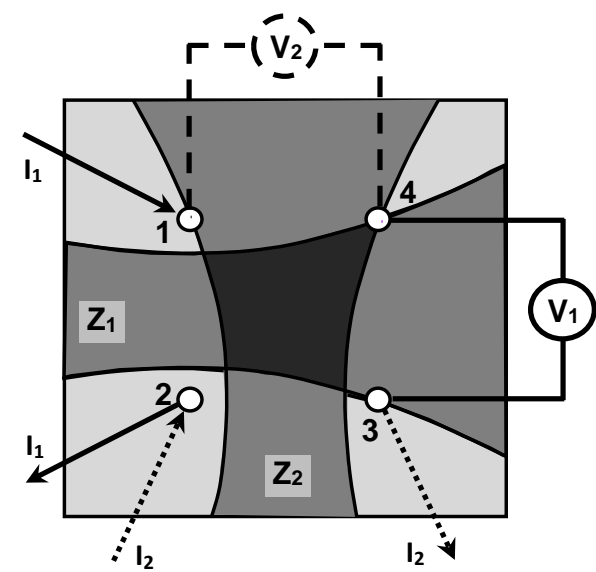

Fig 1. Concept of 4-electrode FIM based on isopotentials

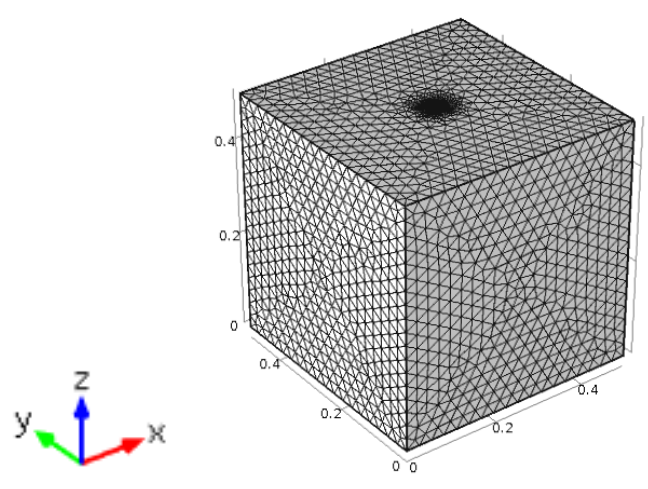

Figure 2: Finite element model of phantom. Size:: $50 \mathrm{~cm} \times 50 \mathrm{~cm} \times 50 \mathrm{~cm}$. Electrodes on one top. $\mathrm{x}, \mathrm{y}, \mathrm{z}$ directions as shown.

However, the negative sensitivities were reduced in the adjacent zones in all the three versions of FIM compared to that in a simple TPIM. This happens since the transfer impedances in the two orthogonal directions are summed in FIM and the small negative sensitivity on one side is cancelled by a small positive sensitivity for the orthogonal configuration. Furthermore, as one goes deeper into the $3^{\text {rd }}$ dimension, the central focused sensitivity becomes predominant and the negative sensitivity becomes negligible. Again, right below the electrodes there are zones of high sensitivity, but this decreases sharply with depth. So the focusing property improves with depth on this count as well.

The present work uses a Finite Element method (FEM) to address the sensitivity situation in a 4-FIM. There are several parameters which affect the transfer impedance of FIM and the effect of these parameters have been studied in detail using a software package named COMSOL which implements FEM. In practice it may be possible to have prior information of several of these parameters so that the effect of only one can be found out from the focused impedance measurements.

The effects of the parameters mentioned above have been studied using COMSOL in the present work 


\section{METHODS}

As mentioned before, we measure transfer impedance in a tetrapolar electrical impedance system. The focused impedance similarly gives transfer impedance and all references to 'impedance' in this paper essentially means 'transfer impedance'

As mentioned before, in 4-FIM an alternating current of constant amplitude is driven through two adjacent pairs and potential is measured across the other two. Then the configuration is rotated by $90^{\circ}$ and again a measurement is taken. Because of the constant current, the potentials are proportional to the respective transfer impedances. The average of the two transfer impedances gives the focused Impedance, FZ.

Although the above concept is developed in $2 \mathrm{D}$, because of current flow in the $3^{\text {rd }}$ dimension, a focusing effect is perceivable at depths, of the order of the electrode spacing. The focusing effect was verified through phantom experiments [Rabbani et al 1999, Rabbani and Karal 2008,] and point response analyses based on lead fields assuming a semi-infinite medium [Islam et al 2010] earlier.

In the first part of the present work we used COMSOL to perform point response studies at different depths with respect to the electrode plane, assuming the electrodes to be at the surface of a bound volume of specific size. For this simulation the adjacent electrode separation was taken as $10 \mathrm{~cm}$ in a cubic phantom containing saline of dimension $50 \mathrm{~cm} \times 50 \mathrm{~cm} \times 50 \mathrm{~cm}$.

In the second part of the present work we performed simulations for spherical objects of finite dimensions sweeping across the focused zone. Some specific values for all the necessary parameters required by the COMSOL software were provided for this purpose. This sweep was again performed at a few depths. The results were compared with that of phantom experiments performed earlier in this laboratory [Rabbani and Karal 2008, Iquebal and Rabbani 2010] and are shown in the next section.

In many applications a target object is immersed in a background with different impedance properties, which may be assumed homogeneous. A 4-FIM system typically attempts to make measurements using skin surface electrodes. There are many variables involved, but if all but one remains fixed, and if apriori knowledge of the others are available, then it should be possible to get information on the target object. Specifically, if the impedivity (from impedance) of the target object changes with time, and if the others do not change then a difference of measurements highlights the target object. Similarly if there is a frequency related change in the target object that is different from the background, again it may be possible to highlight the target object and obtain information regarding the object.

To simulate such a measurement the typical variables are, electrode separation $s$, radius of the spherical target object $r$, resistivity of the homogeneous background $\rho_{b}$, permittivity of the background $\varepsilon_{b}$, resistivity of the target object $\rho_{o}$, permittivity of the object $\varepsilon_{o}$ and depth of object from the electrode plane $d$. The centre of the target object was supposed to be placed at the centre of the focused zone always. In the present study frequency variation was not considered, and the permittivity of the object and the background were kept fixed. Plots were obtained by taking each of the as a variable in turn, while keeping all others fixed.

The above statement may thus be expressed as,

$$
\mathrm{FZ}=f\left(s, r, \rho_{b}, \rho_{o}, d\right) \quad \ldots \quad \ldots
$$

The variation of FZ with each of the variable parameters was studied using COMSOL and the results are presented in the next section. 


\section{RESULTS AND OBSERVATIONS}

Fig. 2 shows finite volume used for this study. It is a cube of size $50 \mathrm{~cm} \times 50 \mathrm{~cm} \times 50 \mathrm{~cm}$ and is assumed to be filled up with a conducting medium of homogeneous conductivity, such as saline.

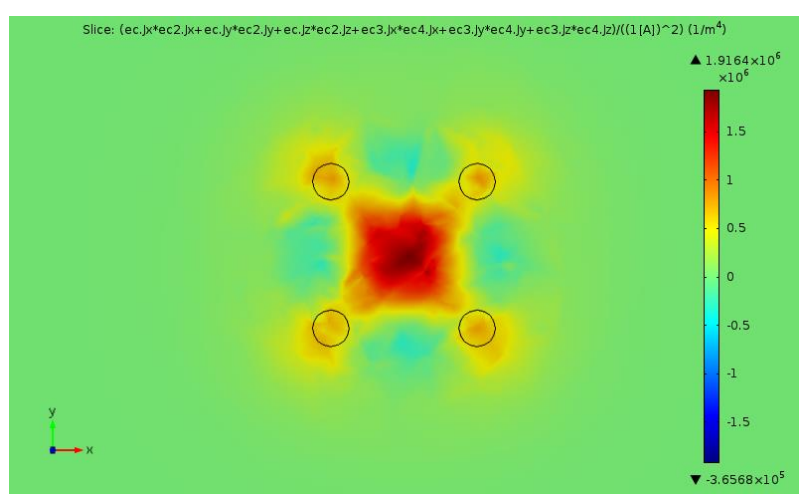

Depth: $0.5 \mathrm{~cm}$

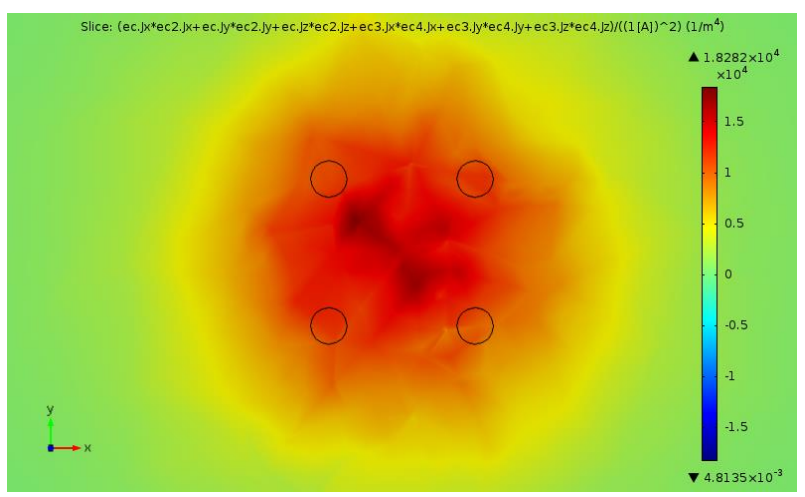

Depth: $3 \mathrm{~cm}$

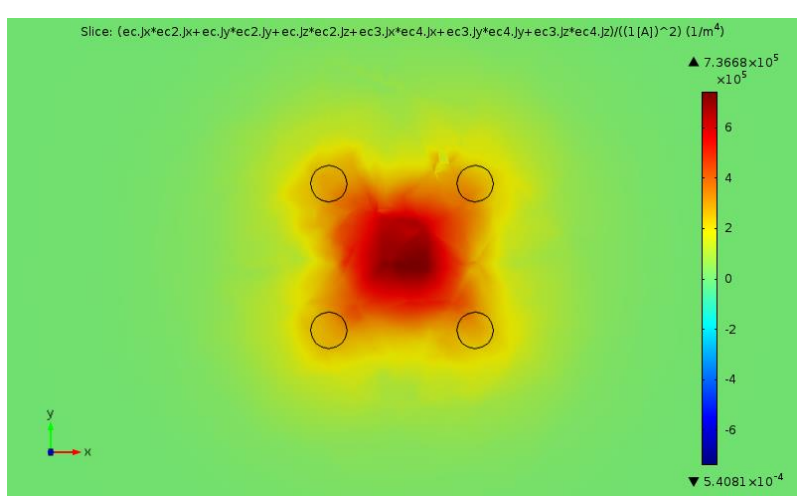

Depth: $1 \mathrm{~cm}$

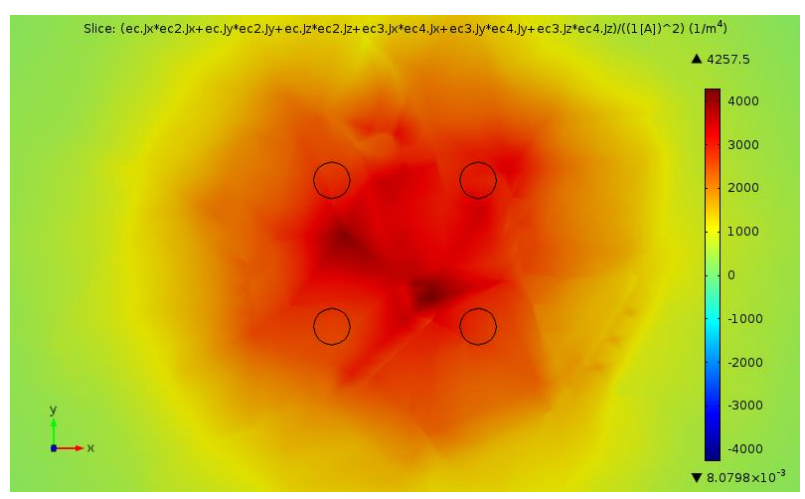

Depth: $4 \mathrm{~cm}$

Figure 3. 2D sensitivity maps at different depths from the electrode plane in a homogeneous medium, obtained using COMSOLE software package employing FEM. Phantom size: $50 \mathrm{cmx} 50 \mathrm{cmx} 50 \mathrm{~cm}$, Electrode separation: $10 \mathrm{~cm}$. Note very small negative sensitivity at $0.5 \mathrm{~cm}$ depth which is totally absent at $1 \mathrm{~cm}$ depth or beyond.

Fig. 3 shows the 2D point sensitivities obtained for this homogenous medium at four depths using an electrode separation of $10 \mathrm{~cm}$. The position of the electrodes are indicated by circles in the figures. The assumed electrode separation, depths and other relevant parameters are shown in the figure caption. The focusing effect is visible; it can be seen that the effects of surrounding regions are very small. A small negative sensitivity exists around the periphery of the focused region for very shallow depths (light blue), but vanishes with depth. At a depth of $1 \mathrm{~cm}(10 \%$ of the adjacent electrode separation) and beyond, negative sensitivity is totally absent. At $0.5 \mathrm{~cm}$ depth, reasonably high sensitivity zones are observed at the four electrodes, as was also observed in reference 3 using lead field analysis. With increase in depth the focused zone expands and encompasses the electrodes, resulting in an almost symmetric focused zone. This is attractive from a practical point of view. 


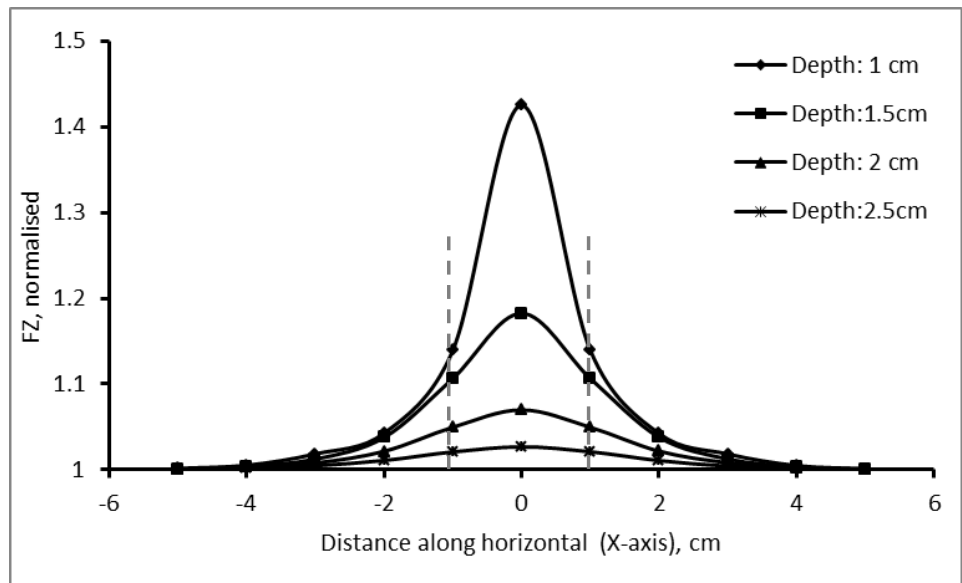

Figure 4: Variation of Focused Impedance FZ, normalised with respect to the background, with object moved through the centre of the square focused region, parallel to one of the sides, for different depths. Background resistivity: $1 \Omega \mathrm{m}$, Object resistivity: $10 \Omega \mathrm{m}$, Electrode separation: $2 \mathrm{~cm}$, Object diameter: $2 \mathrm{~cm}$

For the second part of the experiment for the sweep of a spherical object the adjacent electrode spacing of 4-FIM was $2 \mathrm{~cm}$. The result of sweeping a spherical object of diameter $2 \mathrm{~cm}$ along a direction parallel to the sides along the centre of the focused zone is shown in Figure 4 at a single depth of $1 \mathrm{~cm}$ as indicated in the figure caption. The value of the impedance at the edges of the focused zone, indicated by two straight lines in the figure is considerably lower than the peak value and the value continues to decrease sharply beyond. Therefore, this shows the focusing effect very clearly.

Variations in FZ with individual variables are presented in Figures 5 to 10, with all the details given in the respective figure captions, all for spherical objects with finite dimensions comparable to the electrode separation.

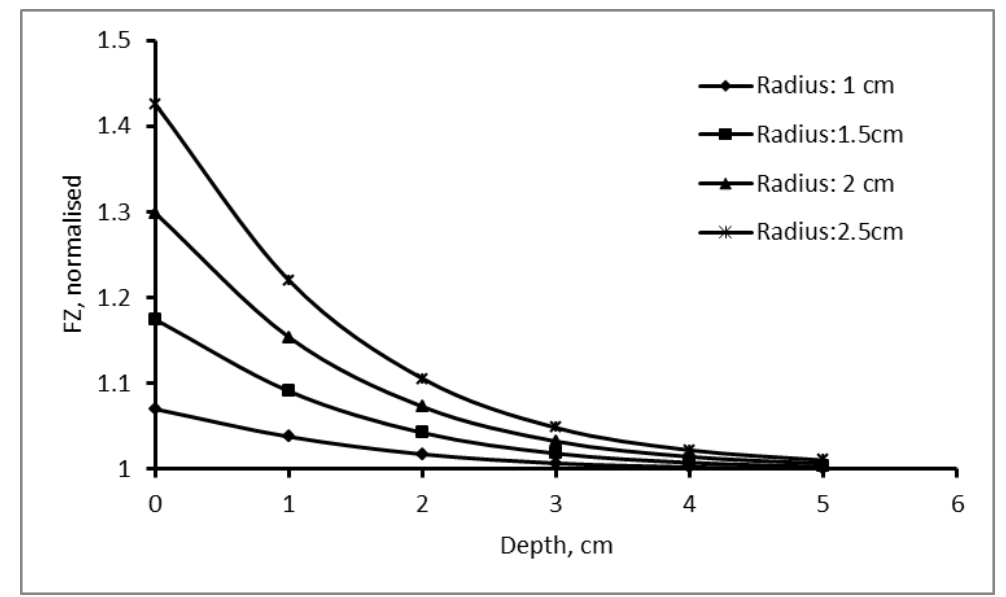

Figure 5: Variation of Focused Impedance FZ, normalised with respect to the background, with depth for objects with different radii, placed at the centre of the focused region. Background resistivity: $1 \Omega \mathrm{m}$, Object resistivity: $10 \Omega \mathrm{m}$, Electrode separation: $2 \mathrm{~cm}$.

Figure 5 shows the impedance values, normalised with respect to the background, as a function of depth. It shows that the focused impedance decreases with depth sharply, gradually going down to the background. It can also be seen that values can be distinguished from the background reasonably well down to a depth of about $2 \mathrm{~cm}$, the same length as the electrode separation. This compares reasonably 
well with the experimental phantom study by Iquebal and Rabbani 2010. Fig. 6 shows the variation with impedance with electrode separation. With an increase in electrode separation for the same object at the same depth FZ decreases; this is because the object takes a lesser percentage of the focused zone. In Figure 7, the FZ value has been normalised to the value obtained with an object of radius $1 \mathrm{~cm}$ (diameter: $2 \mathrm{~cm}$ ), placed centrally in the focused zone at a depth of $4 \mathrm{~cm}$. With an increase in the radius of the object the change in impedance is small till the object radius is about $2 \mathrm{~cm}$ (diameter: $4 \mathrm{~cm}$ ). Beyond this value the impedance increases gradually, a sharp increase is noted beyond a radius of $3.5 \mathrm{~cm}$ (diameter $7 \mathrm{~cm}$ ). Since the equipotential surfaces at the potential measuring electrodes curve away from the centre with depth in the volume the focused zone also increases in dimension. out inside $t$ when the object diameter is of the order of the small with respect to the electrode separation. The depth is also a consideration here. As the object radius becomes bigger than the electrode separation, the rate of change increases. The object depth had to be kept at $4 \mathrm{~cm}$ in this case in order to accommodate an object with a radius of $4 \mathrm{~cm}$. However, the volume of the object should be more relevant in this study which has been shown in Figure 8. Figure 9 shows a very useful situation. It shows the variation with object resistance. In cases like breast tumour, the impedance of a malignant tumour is usually vary small compared to that of a benign tumour at same frequency of measurement. Therefore, this graph will help in interpreting such measurements. The background resistivity is important if one wants a static impedance measurement, and this is shown in Figure 10. Ideally it should have been a straight line for an infinite homogeneous medium. The object and the finite dimension of the medium contributes to the small change observed. Figure 11 shows how the focusing for the same object varies with depth. This needs to be kept in mind while interpreting results for objects at different depths. Some factors may be generated that can provide a compensation to the depth for a particular situation, and may enable a quantitative estimation possible. Figure 12 shows a set of curves which agree well to a similar phantom study performed by Iquebal and Rabbani 2010 .

\section{DISCUSSION}

Two situations need to be highlighted, particularly because of the above mentioned negative sensitivity. In one, a target organ or an object with a bounded geometry may remain within the focused zone, and the interest may lie in looking at changes in this organ only with time or with frequency. If the impedance of the surrounding background volume does not change then the negative sensitivity will be of no concern.

On the other hand, as for lungs, if the target object is large and extends beyond the focused zone, and if a study is targeted within a localized region of this large extended medium, then the negative sensitivity region may be an issue that may not be ignored.

A model of a target measurement system based on finite element Method (FEM) offers a great opportunity in predicting behaviours expected from measurements of electrical impedance using different electrode configurations. COMSOL comes in handy in such an effort. Focused Impedance Method (FIM) with its three versions $-8,6$ and 4 electrodes were initially conceived by one of the authors based on simple equipotential concepts and were verified through phantom experiments. Use of FEM has given the opportunity of fine tuning the concepts together with the possibility of modeling different target organs in the human body. The present effort concentrated on the 4 electrode FIM to verify and improve the understanding of the degree of focusing and the effect of variations due to changes in a number of possible variables. This understanding will help in the designing of experiments to study a particular organ or a region in the human body for physiological 
study and for diagnosis of diseases or disorders. Particularly, applications in the studies of lungs ventilation, stomach emptying, stomach acid secretion, bladder emptying and breast tumour characterization have already been taken up where this numerical modeling may be applied fruitfully.

\section{ACKNOWLEDGEMENTS}

Acknowledgements are due to the International Science Programme (ISP) of Uppsala University, Sweden to provide a major funding for this research. Some funding was also available from the Ministry of Education of the Government of Bangladesh.

\section{REFERENCES}

Islam N, Rabbani K S and Wilson A J, 2010, The sensitivity of focused electrical impedance measurements, Physiol. Meas. 31, S97-S109, doi:10.1088/0967-3334/31/8/S08.

Iquebal A H M and Rabbani K S, 2010, 3D sensitivity of 6-electrode Focused Impedance Method (FIM), J. Phys.: Conf. Ser. 224 (2010)012156 (IOP Publishing) doi:10.1088/1742$6596 / 224 / 1 / 012156$.

Rabbani K S, 2010, Focused Impedance Method (FIM) and Pigeon Hole Imaging (PHI) for localized measurements - a review, J. Phys.: Conf. Ser. 224 (2010) 012003 (IOP Publishing) doi:10.1088/1742-6596/224/1/012003.

Rabbani K S and Kadir M A, 2011, Possible applications of Focused Impedance Method (FIM) in biomedical and other areas of study, Bangladesh Journal of Medical Physics, 4, 67-74.

Rabbani K S and Karal M A S, 2008, A new four-electrode Focused Impedance Measurement (FIM) system for physiological study, Annals of Biomedical Engg (Springer, US), 36, 1072-1077.

Rabbani K S, Sarker M, Akond M H R and Akter T, 1999, Focused Impedance measurement (FIM) A new technique with improved zone localisationin "Electrical Bioimpedance methods", Annals of the New York Academy of Sciences, 873, 408 - 420.

Grimnes S., and Martinsen OG, 2007, Sources of error in tetrapolar impedance measurements on biomaterials and other ionic conductors, J. Phys. D, Appl. Phys., 40, 9-14.

Kwon H, Wi H, Karki B, Lee EJ, McEwan A, Woo EJ, Harrach B and Seo JK, 2012, Bioimpedance spectroscopy tensor probe for anisotropic measurements TI Oh Electronics letters 48, 12531255 